\title{
Isolation of Weissella cibaria from Pacific White Shrimp (Litopenaeus vannamei) Gastrointestinal Tract and Evaluation of Its Pathogenic Bacterial Inhibition
}

\section{Nguyen Duc Huy ${ }^{1, *}$, Le My Tieu Ngoc ${ }^{1}$, Nguyen Hoang Loc ${ }^{2}$, Tran Thuy Lan ${ }^{1}$, Hoang Tan Quang ${ }^{1}$, Tran Quoc Dung ${ }^{3}$, Truong Thi Phuong Lan ${ }^{4}$, Vu Duc Hoang ${ }^{5}$ and Nguyen Thi Dong Phuong ${ }^{6}$}

\author{
1 Institute of Biotechnology, Hue University, Hue, 530000, Vietnam \\ 2Faculty of Biology, University of Science, Hue University, 530000, Vietnam \\ ${ }^{3}$ Faculty of Biology, University of Education, Hue University, 530000, Vietnam \\ ${ }^{4}$ Faculty of Basic Science, Hue University of Medicine and Pharmacy, Hue University, \\ Hue, 530000, Vietnam \\ ${ }^{5}$ Faculty of Biology and Environmental Science, University of Science and Education, \\ The University of Danang, Danang, 550000, Vietnam \\ ${ }^{6}$ Faculty of Chemical Technology and Environment, University of Technology and \\ Education, The University of Danang, Danang, 550000, Vietnam
}

\section{Article Type: Article \\ Article Citation: Nguyen Duc Huy, Le My Tieu Ngoc, Nguyen Hoang Loc, Tran Thuy Lan, Hoang Tan Quang, Tran Quoc Dung, Truong Thi Phuong Lan, Vu Duc Hoang, Nguyen Thi Dong Phuong. Isolation of Weissella cibaria from Pacific white shrimp (Litopenaeus vannamei) gastrointestinal Tract and evaluation of its pathogenic bacterial inhibition. Indian Journal of Science and Technology. 2020; 13(10), 1200-1212. D0l: 10.17485/ijst/2020/ v013i10/149934}

Received date: January 24, 2020 Accepted date: February 22, 2020

*Author for correspondence: Nguyen Duc Huy - ndhuy@hueuni. edu.vn 9 Institute of Biotechnology, Hue University, Hue, 530000, Vietnam

\begin{abstract}
Objective: The present study reported the isolation of lactic acid bacteria from the shrimp gastrointestinal tract and evaluation of its antagonistic activity against pathogenic bacteria. Methods/ statistical analysis: Lactic bacteria from the gastrointestinal tract of alive healthy shrimp were screened on sterilized MRS medium. Vibrio sp. inhibition by isolates was conducted by the agar-well diffusion assay. The isolates were identified by $16 \mathrm{~S}$ rRNA sequencing. Antibacterial activity was investigated against 25 Vibrio sp. isolates, Staphylococcus aureus ATCC 25023, and Escherichia coli ATCC 85922. Hemolytic activity, salt, and antibiotic resistance were also evaluated. Findings: Total of 22 isolates exhibited Vibrio parahaemolyticus inhibition with antagonistic activities ranged from $220 \mathrm{AU} / \mathrm{ml}$ to $460 \mathrm{AU} / \mathrm{ml}$. Sequence analysis of $16 \mathrm{~S}$ rRNA indicated the 5 isolates belong to Weissella cibaria species, namely HN02, HN03, HN04, and HN06 AND HN07. Among these, W. cibaria HN05 had highest antagonistic activity and exhibited the broad inhibition spectrum against Vibrio sp. as well as E. coli ATCC 85922 but not S. aureus ATCC 25023. $\beta$-hemolytic and salt resistance assays showed that $W$. cibaria $\mathrm{HN} 05$ had no $\beta$-hemolytic activity and grew well in medium containing $5 \%$ of salt. Antibiotic resistance analysis indicated $W$. cibaria HN05 was suppressed by ampicillin, chloramphenicol, oxytetracycline hydrochloride, and chlortetracycline hydrochloride. Application/improvements: This is the first study on isolation and
\end{abstract}


Nguyen Duc Huy, Le My Tieu Ngoc, Nguyen Hoang Loc, Tran Thuy Lan, Hoang Tan Quang, Tran Quoc Dung, Truong Thi Phuong Lan, Vu Duc Hoang and Nguyen Thi Dong Phuong

evaluation pathogenic bacteria including Vibrio sp. inhibition by $W$. cibaria, which is isolated from shrimp gastrointestinal tract.

Keywords: Antagonistic Activity, Gastrointestinal Tract, Shirmp, Weissella cibaria, Vibrio sp.

\section{Introduction}

Pacific white shrimp, Litopenaeus vannamei, is the most popular shrimp species due to their survival advantages such as fast growth rate and strong adaptability to the environment [1]. The shrimp is widely cultivated in Southeast Asia, China, India, the United States of America, Mexico, and Latin America, yielding more than 4 million tons in 2016 [2]. However, high frequency of diseases has severely influenced the shrimp farming [1].

In 2009, acute hepatopancreatic necrosis disease (AHPND) was first reported in China then disease outbreaks occurred in various countries in Southeast Asia and America, resulting in up to $100 \%$ mortality. Vibrio species such as $V$. parahaemolyticus and $V$. harveyi were demonstrated as causative agent of AHPND [3-4]. Meanwhile, Vibrio sp. also response for disease on shrimp including luminous vibriosis, tail necrosis, shell disease, red disease, loose shell syndrome, white gut disease, white spot, etc [5-7]. It is estimated shrimp farming industry lost more than $1 \$$ billion US dollar per year [8]. Thus, developing an appropriate strategy to control Vibrio species in aquaculture not only increases the productivity but also enhance the product safety.

The infectious disease prevention and control in aquaculture using commercial antibiotics is still the chosen of many farmers due to high inhibiting ability to pathogens. However, overuse of antibiotics can lead to the bloom of drug-resistant bacteria as well as affect aquatic environment and further to human [9-10]. Naturally, Vibrio pathogen can be inhibited by other bacteria which are able to secrete anti-bacterial substances during their growth. The bacteria show beneficial effect on the host is called as probiotics and mainly belong to the lactic acid bacteria such as Bacillus, Lactobacillus, Bifidobacterium, Streptococcus, etc [11-12]. Probiotic bacteria strengthen the epithelial barrier, increase adhesion to the intestinal mucosa, inhibit simultaneously pathogens, eliminate competition for pathogenic microorganisms and produce antimicrobial substances as well as modulate of the immune system [13]. Many probiotic bacteria are currently being investigated and applied to aquaculture, especially shrimp farming [14-17]. Thus, the use of probiotics for disease prevention and nutrition improvement in shrimp aquaculture is becoming increasingly popular due to an increasing demand for environment-friendly aquaculture [9].

Weissella cibaria is a Gram-positive and obligate heterofermentative lactic acid bacteria [18]. It is known to produce native substances, including antimicrobial and antifungal agents against Gram-positive bacteria [19], periodontal disease-causing bacteria [20]. Up to date, $W$. cibaria has been used as a probiotic bacterium in different fermented products such as Kimchi [21], cottage cheese [22] Chinese jiang-shui [23], and shrimp paste [24]. $W$. cibaria M3 strain can reduce the fermentation time along with enhancing flavor and 
product quality of Chouguiyu, a Chinese traditional fermented fish [25]. Thus, this strain has been potential attracted to use as probiotic agent [26].

\section{Material and Methods}

\subsection{Sampling and Lactic Acid Bacteria Screening}

The alive healthy shrimp were collected from local shrimp farms at Thua Thien Hue province, Vietnam and delivered to the laboratory. Digestive tracts were collected and dissolved in $500 \mu \mathrm{l}$ of $0.9 \% \mathrm{NaCl}$ solution. Two hundred microliters were inoculated in $5 \mathrm{ml}$ of sterilized Man Rogosa Sharpe (MRS) and incubated at $30{ }^{\circ} \mathrm{C}$ for $24 \mathrm{~h}$ without shaking. One hundred microliters were spread onto MRS supplemented with $0.5 \% \mathrm{CaCO}_{3}$ agar medium and cultured at $30{ }^{\circ} \mathrm{C}$ for $24 \mathrm{~h}$. The single colony showing halo zone was picked up and subcultured into new MRS agar plate. The culture was repeated five times to select pure colonies.

\subsection{Screening for Vibrio parahaemolyticus Inhibition Ability}

The potential Vibrio sp. inhibition by isolates was conducted by the agar well diffusion assay using pure isolate collection against $V$. parahaemolyticus on LB agar (1\% tryptone, $0.5 \%$ yeast extract, $1 \% \mathrm{NaCl}$, and $0.8 \%$ agar) medium [27]. A V. parahaemolyticus strain was chosen from Vibrio collection storing at Institute of Biotechnology, Hue University, Vietnam and was cultured in $5 \mathrm{ml}$ of LB medium overnight. Meanwhile, each potential Vibrio sp. inhibition isolate was cultured in $5 \mathrm{ml}$ MRS medium with $180 \mathrm{rmp}$ of shaking, $30{ }^{\circ} \mathrm{C}$ for overnight. Then, the cell density of both overnight cultures was measured spectrophotometrically at $600 \mathrm{~nm}$ wavelength and diluted to final concentration of $10^{8}$ $\mathrm{CFU} / \mathrm{ml}$. Fifty microliters of diluted V. parahaemolyticus culture was spread on LB agar medium, while fifty microliters free-cell diluted supernatant culture of isolate was dropped into $6 \mathrm{~mm}$ hole made on plate. The plate was incubated at $4{ }^{\circ} \mathrm{C}$ for $15 \mathrm{~min}$ then transferred into $30^{\circ} \mathrm{C}$ incubator for $24 \mathrm{~h}$. The diameter of the zone of clearance (DZC) was measured. The antagonistic activity (AU/ml) was calculated by the following formula [28]:

$$
\mathrm{AU} / \mathrm{ml}=\frac{\text { Diameter of the zone of clearance }(\mathrm{mm}) \times 1000}{\text { Volume taken in the well }(\mu \mathrm{l})}
$$

e Vibrio inhibiting capacity was defined based on the antagonistic activity value whereas no inhibition is equal with $\mathrm{AU}=0$, weak inhibition is equal with $0<\mathrm{AU} \leq 200$, moderate inhibition is equal with $200<\mathrm{AU} \leq 280$, and strong inhibition is equal with $\mathrm{AU}>280$.

\subsection{Biochemical and Molecular Identification}

The isolate exhibited V. parahaemolyticus antagonistic activity was stained by Gram's method [29], was conducted catalase assay [29], and was performed microscopic observation at magnification of $100 \times$ (Nikon eclipse 55i, Japan). Molecular identification 
Nguyen Duc Huy, Le My Tieu Ngoc, Nguyen Hoang Loc, Tran Thuy Lan, Hoang Tan Quang, Tran Quoc Dung, Truong Thi Phuong Lan, Vu Duc Hoang and Nguyen Thi Dong Phuong

of isolate was carried out by sequencing the $16 \mathrm{~S}$ rRNA fragment. Briefly, the bacterium was cultured in $5 \mathrm{ml}$ of MRS medium at $30^{\circ} \mathrm{C}$ with a shaking speed of $180 \mathrm{rpm}$ overnight. The cell biomass was harvested by centrifugation at $8000 \mathrm{rmp}$ for $5 \mathrm{~min}$. Total genomic DNA was isolated using CTAB buffer as described by Sambrook [30]. The DNA was qualified on $0.8 \%$ agarose gel and used as temple to amplify the $16 \mathrm{~S}$ rRNA sequence with pair primer set of 27F (5'-AGAGTTTGATCCTGGCTCAG-3') and 1492R (5' GGTTACCTTGTTACGACTT-3'). The PCR product was sequenced (Firstbase, Malaysia) and aligned against GenBank database. The strain identity was defined based on the highest nucleotide sequence similarity. The phylogenetic tree was analyzed using Mega X software based on Maximum likelihood with Booststrap of 500 replicates [31].

\subsection{Evaluation Vibrio sp. Inhibition}

To evaluate the Vibrio sp. inhibition capacity, the selected isolates exhibited $V$. parahaemolyticus inhibition were examined on Vibrio sp. collection storing at Institute of Biotechnology, Hue University, Vietnam. The Vibrio sp. collection was isolated from shrimp being infected by acute hepatopancreatic necrosis disease. The evaluation was carried out using agar well diffusion assay as mentioned above. The antagonistic activity was recorded and evaluated.

\subsection{Evaluation Antibacterial Against Positive and Negative Bacteria}

Staphylococcus aureus ATCC 25023 and Escherichia coli ATCC 85922 (Microbiologics, USA) were used as standard for positive and negative bacteria model, respectively. The evaluation was performed by agar well diffusion assay as mentioned above. The DZC around the hole was recorded and converted into antagonistic activity unit.

\subsection{Salt Resistance Assay}

The single colony of each isolate was cultured in $5 \mathrm{ml} \mathrm{MRS} \mathrm{medium} \mathrm{for} 24 \mathrm{~h}$ at $37^{\circ} \mathrm{C}$. Cells biomass was harvested by centrifugation at $8000 \mathrm{rpm}$ for $5 \mathrm{~min}$ at $4{ }^{\circ} \mathrm{C}$. The cells were resuspended in $\mathrm{NaCl}$ solution (range from $0 \%$ to $25 \%$ ) to final $\mathrm{OD}_{600}$ of 1.0. One hundred microliters of suspension were transferred into nine hundred microliters MRS medium containing the corresponding $\mathrm{NaCl}$ concentration. $\mathrm{OD}_{600}$ was observed before and after $48 \mathrm{~h}$ incubation at $37^{\circ} \mathrm{C}$.

\subsection{Hemolytic Activity}

The selected isolates were subjected on agar plate containing 5\% sheep blood. The overnight supernatant culture in MRS medium was harvested by centrifugation at $8000 \mathrm{rpm}$ for 14 min at $4{ }^{\circ} \mathrm{C}$. Fifty microliters were added in the $6 \mathrm{~mm}$ diameter hole on blood agar plate. The plate was incubated at $37^{\circ} \mathrm{C}$ for $24 \mathrm{~h}$. Hemolytic activity was accessed as clearance zone [32]. 


\subsection{Antibiotic Resistance Assay}

The antibiotic resistance ability of selected isolates was conducted by inoculation the single colony of each isolate on $5 \mathrm{ml}$ MRS medium supplemented with antibiotics (sulfapiridine, sulfathiazole, sulfamethoxazole, oxytetracyclin hydrochloride, chlotetracyclin hydrochloride, ampicillin, and chloramphenicol (Sigma, USA) and kanamycin (Biobasic, Canada)) at $37^{\circ} \mathrm{C}$ for $24 \mathrm{~h}$. The antibiotic concentration was ranged from $25 \mu \mathrm{g} / \mathrm{ml}$ to 500 $\mu \mathrm{g} / \mathrm{ml}$. The cell density was taken by measuring at $\mathrm{OD}_{600}$.

\section{Results and Discussion}

\subsection{Isolation and Identification of Vibrio parahaemolyticus Inhibiting Bacteria}

A total of 23 potential lactic acid bacteria colonies were picked up and purified with serial subculture in which 22 of isolates showed V.parahaemolyticus inhibition at different levels (Figure 1). The antagonistic activities ranged from $220 \mathrm{AU} / \mathrm{ml}$ to $460 \mathrm{AU} / \mathrm{ml}$. Among them, 16 isolates exhibited strong antagonistic effect with activity over 280 units (Table 1). The isolates were then assigned as HN01 to HN22.

The analyses showed 18 isolates were Gram-positive bacteria and only 2 of them exhibited catalase activity (Table 1). From above results, isolates displayed as Grampositive bacteria and had the $V$. parahaemolyticus strong inhibition were identified by the 16S RNA fragment sequencing method. DNA sequence alignment confirmed 5 isolates (HN02, HN03, HN04, HN05, and HN07) have 100\% similarity to Weissella cibaria species and they were deposited on Genbank database as accession number of MK989999, MK990000, MK990001, MK990002, and MK990004, respectively. The phylogenetic tree of strains belongs to W. cibaria including HN02, HN03, HN04, HN05, and HN07 is shown in Figure 2.

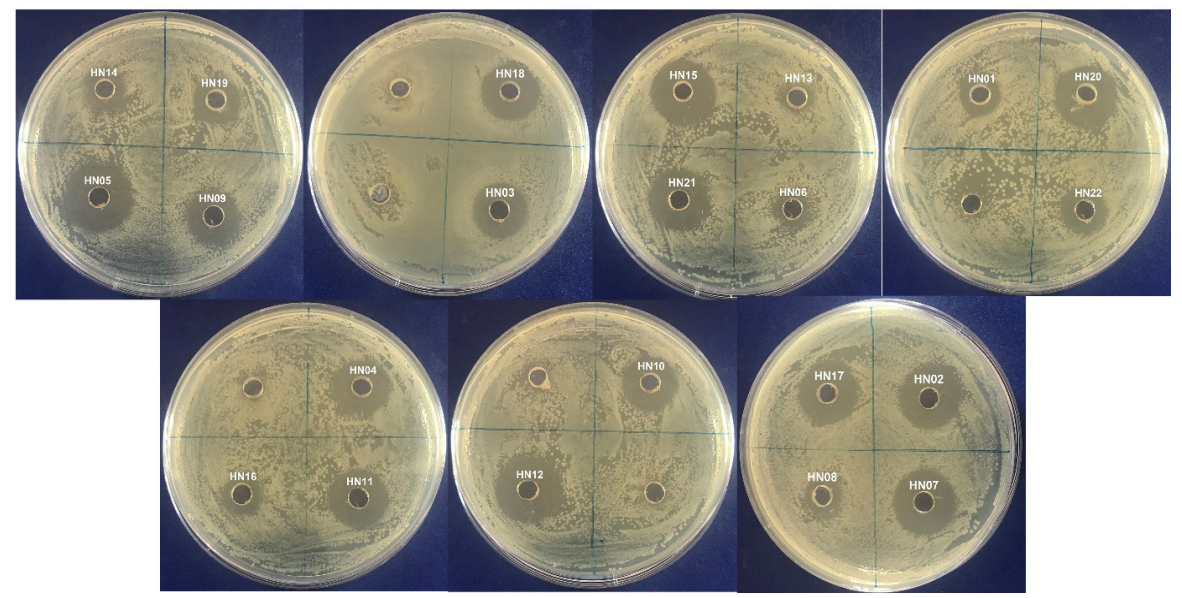

FIGURE 1. Antagonistic activity of isolates against V. parahaemolyticus. 
Nguyen Duc Huy, Le My Tieu Ngoc, Nguyen Hoang Loc, Tran Thuy Lan, Hoang Tan Quang, Tran Quoc Dung, Truong Thi Phuong Lan, Vu Duc Hoang and Nguyen Thi Dong Phuong

TABLE 1. Isolate collection exhibited V. parahaemolyticus inhibition

\begin{tabular}{ccccc}
\hline No. & Strain name & $\begin{array}{c}\text { Antagonistic activity } \\
\text { (AU/ml) }\end{array}$ & Gram profile & Catalase profile \\
\hline 1 & HN01 & 240 & + & - \\
2 & HN02 & 420 & + & - \\
3 & HN03 & 340 & + & - \\
4 & HN04 & 400 & + & - \\
5 & HN05 & 440 & + & - \\
6 & HN06 & 220 & + & - \\
7 & HN07 & 420 & + & - \\
8 & HN08 & 220 & + & - \\
9 & HN09 & 320 & + & - \\
10 & HN10 & 360 & + & - \\
11 & HN11 & 380 & + & - \\
12 & HN12 & 460 & - & - \\
13 & HN13 & 220 & + & - \\
14 & HN14 & 300 & - & - \\
15 & HN15 & 420 & - & - \\
16 & HN16 & 260 & + & - \\
17 & HN17 & 440 & - & + \\
18 & HN18 & 400 & + & - \\
19 & HN19 & 280 & + & - \\
20 & HN20 & 420 & + & \\
21 & HN21 & 400 & + & - \\
22 & HN22 & 300 & & - \\
\hline
\end{tabular}

The antagonistic activity is presented as average data of three replicates.

Gram profile: positive gram $(+)$, negative gram $(-)$.

Catalase profile: catalase activity $(+)$, none catalase activity $(-)$.

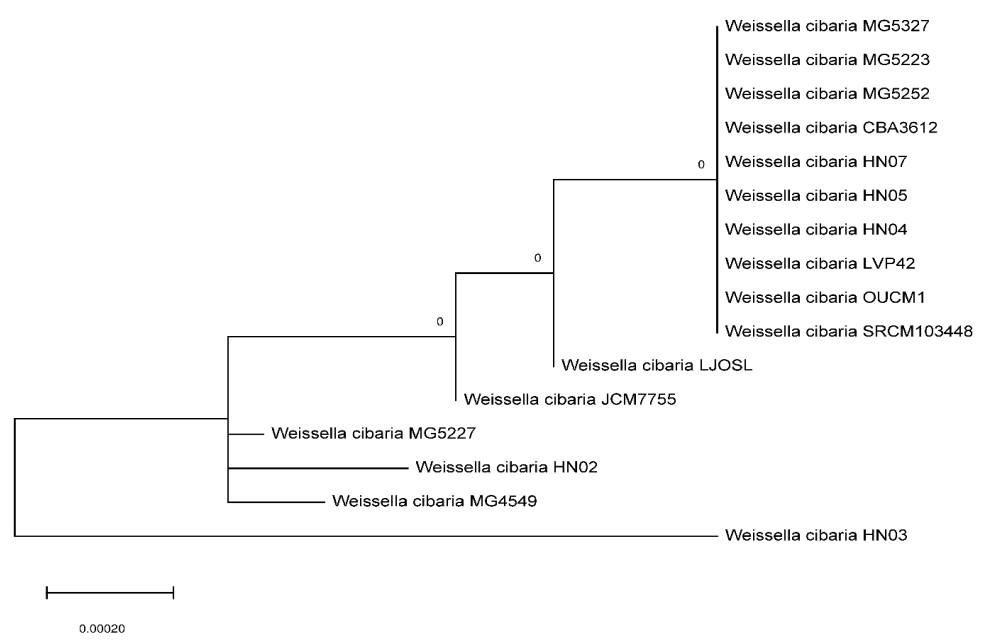

FIGURE 2. Phylogenetic tree among of W. cibaria HN02, W. cibaria HN03, W. cibaria HN04, W. cibaria HN05, W. cibaria HN07, and other W. cibaria. 
Probiotic bacteria are considered as potential approach for sustainable aquaculture. Numerous reports have shown supplementation of probiotic bacteria reduce the number of opportunistic pathogenic bacteria, enhance the resistance against pathogenic bacteria as well as increase the immune response, weight gain rate, survival rate [16-17, 33]. The source probiotic bacteria are various such as fermented food [16], animal milk [28], digestive tract, or gut $[17,34]$. The present study aims to isolate gut bacteria with potential probiotic against pathogenic Vibrio. A collection of 22 isolates from shrimp gastrointestinal tract inhibited V. parahaemolyticus, in which 16 isolates exhibited strong antagonistic activity. The results confirm gastrointestinal tract is a good source to isolate potential probiotic lactic acid bacteria.

The isolate collection consists of both gram-positive and gram-negative bacteria. This finding is in accordance with previous reports showed Vibrio sp. can be inhibited by grampositive and gram-negative bacteria [35, 36]. However, due to most of probiotic bacteria applying in aquaculture is gram-positive such as bacillus, lactobacillus,... [36], thus, the gram-positive bacteria are being selected for further study.

The molecular identification showed that isolates are divided into two group Lactococcus and Weissella genus (data not shown). Dalmacio et al (2011) identified the lactic acid bacteria from fermented shrimp paste by PCR-denaturing gradient gel electrophoresis approach. The author found W. cibaria is among of dominant species [24]. Moreover, Leuconostocaceae family where $W$. cibaria belonging, is particular presented in bacteria community associated with shrimp culture system [37]. However, there is no report on identification W. cibaria in shirmp gut microbiota up to date.

\subsection{Investigation the Board Antibacterial of Selected Isolate}

As shown in Table 2, W. cibaria HN05 exhibited the strongest antagonistic activity against $V$. parahaemolyticus, thus the strain was selected for further investigations. To investigate the ability for a board Vibrio sp. inhibition, W. cibaria $\mathrm{H} 05$ was tested against 25 different strains of Vibrio species. W. cibaria H05 inhibited all tested Vibrio sp. with antagonistic activity ranged from $220 \mathrm{AU} / \mathrm{ml}$ to $500 \mathrm{AU} / \mathrm{ml}$. Interestingly, W. cibaria $\mathrm{H} 05$ strongly inhibited 22 Vibrio species with antagonistic activity $>280 \mathrm{AU} / \mathrm{ml}$.

The results on testing against E. coli ATCC 85922 and S. aureus ATCC 25023 indicated that $W$. cibaria $\mathrm{HN} 05$ inhibited $E$. coli growth with antagonistic activity of $240 \mathrm{AU} / \mathrm{ml}$ but no S. aureus ATCC 25023 inhibition activity was observed.

Our study is the first time described vibrios inhibition activity by the W. cibaria which is isolated from shrimp sources. Interestingly, W. cibaria isolates exhibited strong antagonistic activity against Vibrio sp. up to $500 \mathrm{AU} / \mathrm{ml}$ as well as E. coli but limited to $S$. aureus. It is well known that $W$. cibaria belongs to probiotic group. W. cibaria secretes antibacteria substances such as bacteriocin [18], or modules immune response [38] or inhibits the colonization of pathogenic bacteria [39]. Thus, isolate W. cibaria HN05 could be good probiotic candidate for shrimp feed. 
Nguyen Duc Huy, Le My Tieu Ngoc, Nguyen Hoang Loc, Tran Thuy Lan, Hoang Tan Quang, Tran Quoc Dung, Truong Thi Phuong Lan, Vu Duc Hoang and Nguyen Thi Dong Phuong

TABLE 2. Antagonistic activity of W. cibaria HN05 against Vibrio sp.

\begin{tabular}{ccc}
\hline No. & Name of Vibrio sp. & $\begin{array}{c}\text { Antagonistic activity } \\
\text { (AU/ml) }\end{array}$ \\
\hline 1 & VTVV4(3) & 400 \\
2 & VTVV2(8) & 200 \\
3 & VTVV3(3) & 420 \\
4 & VTVX3a(13) & 300 \\
5 & VTVX2a(11) & 260 \\
6 & VTVV4(1) & 240 \\
7 & VTVV1(6) & 320 \\
8 & VTVX1a(9) & 200 \\
9 & VTVX4a & 220 \\
10 & VC1 & 160 \\
11 & VTVV4(4) & 400 \\
12 & VC13 & 240 \\
13 & VTVV2(7) & 440 \\
14 & VTVX4b & 360 \\
15 & VC7 & 340 \\
16 & VTVV1(5) & 300 \\
17 & VC1 & 400 \\
18 & VTVX1b(10) & 360 \\
19 & VC13 & 320 \\
20 & VTVX2b(12) & 360 \\
21 & VC14 & 300 \\
22 & VTVX3b(14) & 300 \\
23 & VC9 & 320 \\
24 & VC12 & 340 \\
25 & VC5 & 300 \\
\hline
\end{tabular}

The antagonistic activity is presented as average data of three replicates.

\subsection{Salt Resistance and Hemolytic Activity}

To evaluate salt resistance, $W$. cibaria $\mathrm{HN} 05$ was cultured in medium containing $\mathrm{NaCl}$ range from $0 \%$ to $20 \%$. Overall, increasing $\mathrm{NaCl}$ concentration dramatically reduced $W$. cibaria HN05 growth. W. cibaria $\mathrm{HN} 05$ grew well in the presence of $\mathrm{NaCl}$ concentration up to 5 $\%$. However, W. cibaria HN05 completely inhibited when increasing $\mathrm{NaCl}$ concentration above $10 \%$ (Figure 3).

Figure 4 showed the hemolytic activity of W. cibaria HN05 on sheep blood agar plate. The results indicated S. aureus ATCC 25023 exhibited the $\beta$-hemolytic activity whereas no $\beta$-hemolytic activity observed in the culture of $W$. cibaria HN05.

The extracellular substances of $W$. cibaria HN05 did not show the $\beta$-hemolytic (Figure 4). Our finding is in accordance to report by [32] where is no $\beta$-hemolytic observation. Salt resistance is one of importance factors for probiotic applying in shrimp cultivation which salt concentration in water is usually $10 \%$ or higher. W. cibaria HN05 grew well in the medium containing $5 \%$ salinity, resulting in advantage when supplement to shrimp pond culture. 


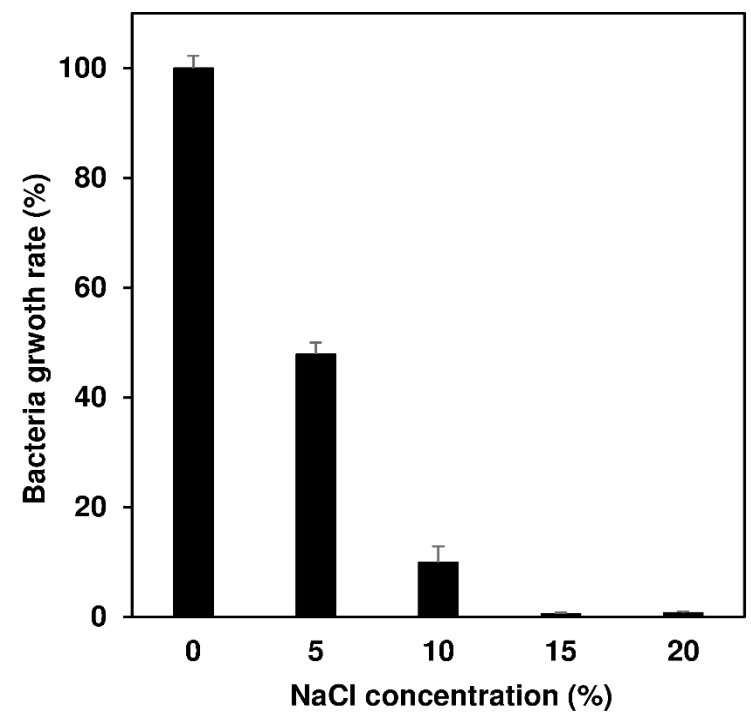

FIGURE 3. $\mathrm{NaCl}$ resistance ability of W. cibaria HN05.

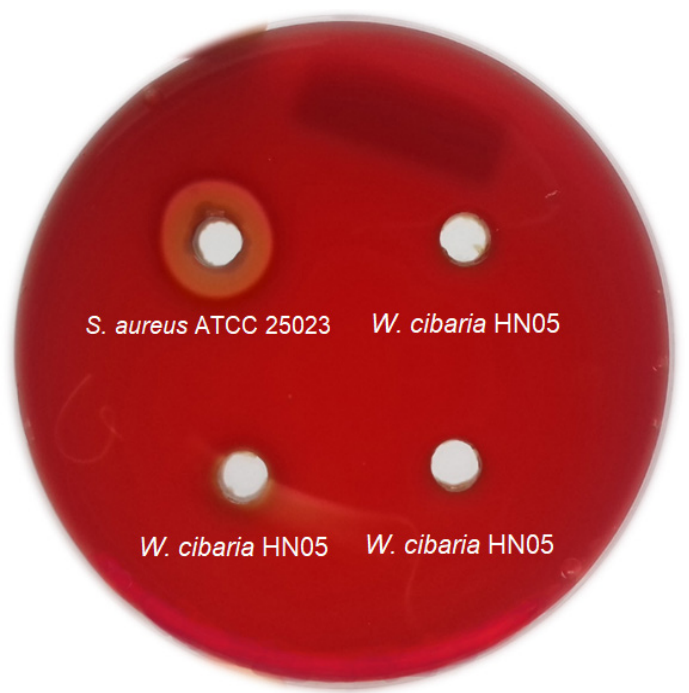

FIGURE 4. Hemolytic activity of W. cibaria HN05 and S. aureus ATCC 25023.

\subsection{Antibiotic Resistance}

The antibiotic resistance tests showed that W. cibaria HN05 grew well in the presence of sulfapyridine, sulfathiazole, sulfamethoxazole, and kanamycin. Oxytetracycline hydrochloride and chlortetracycline hydrochloride inhibited $W$. cibaria HN05 at concentration $\geq 100 \mu \mathrm{g} / \mathrm{ml}$. Ampicillin and chloramphenicol completely inhibited $W$. cibaria HN05 at the concentration of $25 \mu \mathrm{g} / \mathrm{ml}$ (Table 3). In the study on safety evaluation of probiotic W. cibaria, the strain W. cibaria CMU exhibited strong resistant to kanamycin, 
Nguyen Duc Huy, Le My Tieu Ngoc, Nguyen Hoang Loc, Tran Thuy Lan, Hoang Tan Quang, Tran Quoc Dung, Truong Thi Phuong Lan, Vu Duc Hoang and Nguyen Thi Dong Phuong

vancomycin while was weak against ampicillin, oxytetracycline, tetracycline, etc [32]. In the present study, W. cibaria HN05 exhibited resistance to kanamycin and sulfonamides group in vitro.

TABLE 3. Antibiotic resistance of W. cibaria HN05

\begin{tabular}{llcccc}
\hline \multirow{2}{*}{ Antibiotic } & \multicolumn{5}{c}{ Concentration $(\boldsymbol{\mu g} / \mathrm{ml})$} \\
\cline { 2 - 6 } & $\mathbf{2 5}$ & $\mathbf{5 0}$ & $\mathbf{1 0 0}$ & $\mathbf{2 5 0}$ & $\mathbf{5 0 0}$ \\
\hline Sulfapyridine & & & & & \\
Sulfathiazole & & & & & \\
Sulfamethoxazole & & & - & - & - \\
Oxytetracycline hydrochloride & & & - & - & - \\
Chlortetracycline hydrochloride & & - & - & - & - \\
Ampicillin & - & - & & & \\
Kanamycin & - & - & - & - & - \\
Chloramphenicol & - & - & & &
\end{tabular}

-: not growth.

\section{Conclusion}

W. cibaria was isolated for the first time from shrimp digestive tract. The isolate shows a strong ability to inhibit various pathogens, including Vibrio sp. as well as E. coli. W. cibaria resists to salt at concentration usually higher than that required for shrimp growth and exhibits no $\beta$-hemolytic activity. The isolate could be a potential probiotic for shrimp in order to reduce the infection of pathogen bacteria in shrimp intestinal. Further, in vivo study is needed to evaluate the probiotic effect of isolate fully.

\section{Conflicts of Interest}

The authors declare no conflict of interest.

\section{Acknowledgment}

The study received funding support from Ministry of Education and Training under grant number CT-2018-DHH-06.

\section{References}

1. Li J, Jiang H, Li L, Zhang X, Chen J. The effect of disease and season to hepatopancreas and intestinal mycobiota of Litopenaeus vannamei. Frontiersin Microbiology. 2019, 10, 889. DOI: 10.3389/fmicb.2019.00889.

2. FAO. Cultured aquatic species information programme: Penaeus vannamei (Boone, 1931). http://www.fao.org/fishery/culturedspecies/Penaeus_vannamei/en. Date accessed: 01/10/2020. 
3. De Schryver P, Defoirdt T, Sorgeloos P. Early mortality syndrome outbreaks: a microbial management issue in shrimp farming? PLoS Pathogens. 2014, 10(4), e1003919. DOI: 10.1371/ journal.ppat.1003919.

4. de la Pena LD, Cabillon NA, Catedral DD, Amar EC, Usero RC, Monotilla WD,Calpe AT, Fernandez DD, Saloma CP. Acute hepatopancreatic necrosis disease (AHPND) outbreaks in Penaeus vannamei and P. monodon cultured in the Philippines. Diseases of Aquatic Organisms. 2015, 116(3), 251-254. DOI: 10.3354/dao02919.

5. Jayasree L, Janakiram P, Madhavi R. Characterization of Vibrio spp. associated with diseased shrimp from culture ponds of Andhra Pradesh (India). Journal of the World Aquaculture Society. 2006, 37(4), 523-532. DOI: 10.1111/j.1749-7345.2006.00066.x.

6. Austin B. Vibrios as causal agents of zoonoses. Veterinary Microbiology. 2010, 140(3-4), 310317. DOI: 10.1016/j.vetmic.2009.03.015.

7. Wang L, Chen Y, Huang H, Huang Z, Chen H, Sha Z. Isolation and identification of Vibrio campbellii as a bacterial pathogen for luminous vibriosis of Litopenaeus vannamei. Aquaculture Research. 2015, 46(2), 395-404. DOI: 10.1111/are.12191.

8. Lee CT, Chen IT, Yang YT, Ko TP, Huang YT, Huang JY, Huang MF, Lin SJ, Chen CY, Lin SS, Lightner DV, Wang HC, Wang AH, Wang HC, Hor LI, Lo CF. The opportunistic marine pathogen Vibrio parahaemolyticus becomes virulent by acquiring a plasmid that expresses a deadly toxin. Proceedings of the National Academy of Sciences of the United States of America. 2015, 112(34), 10798-10803. DOI: 10.1073/pnas.1503129112.

9. Ninawe AS, Selvin J. Probiotics in shrimp aquaculture: avenues and challenges. Critical Reviews in Microbiology. 2009, 35(1), 43-66. DOI: 10.1080/10408410802667202.

10. Kummerer K. Antibiotics in the aquatic environment - a review - part I. Chemosphere. 2009, 75 (4), 417-434. DOI: 10.1016/j.chemosphere.2008.11.086.

11. Verschuere L, Rombaut G, Sorgeloos P, Verstraete W. Probiotic bacteria as biological control agents in aquaculture. Microbiololy and Molecular Biology Reviews. 2000, 64(4), 655-671. DOI: 10.1128/MMBR.64.4.655-671.2000.

12. Tan LT, Chan KG, Lee LH, Goh BH. Streptomyces bacteria as potential probiotics in aquaculture. Frontiers in Microbiology. 2016, 7, 79. DOI: 10.3389/fmicb.2016.00079.

13. Kavitha M, Raja M, Perumal P. Evaluation of probiotic potential of Bacillus spp. isolated from the digestive tract of freshwater fish Labeo calbasu (Hamilton, 1822). Aquaculture Reports. 2018, 11, 59-69. DOI: 10.1016/j.aqrep.2018.07.001.

14. Xie JJ, Liu QQ, Liao S, Fang HH, Yin P, Xie SW, Tian LX, Liu YJ, Niu J. Effects of dietary mixed probiotics on growth, non-specific immunity, intestinal morphology and microbiota of juvenile pacific white shrimp, Litopenaeus vannamei. Fish and Shellish Immunology. 2019, 90, 456-465. DOI: 10.1016/j.fsi.2019.04.301.

15. Nguyen HT, Nguyen TT, Pham HTT, Nguyen QTN, Tran MT, Nguyen AH,Phan TN, Bui HTV, Dao HTT, Nguyen ATV. Fate of carotenoid-producing Bacillus aquimaris SH6 colour spores in shrimp gut and their dose-dependent probiotic activities. PLoS One. 2018, 13(12), e0209341. DOI: 10.1371/journal.pone.0209341.

16. Wang YC, Hu SY, Chiu CS, Liu CH. Multiple-strain probiotics appear to be more effective in improving the growth performance and health status of white shrimp, Litopenaeus vannamei, than single probiotic strains. Fish and Shellfish Immunology. 2019, 84, 1050-1058. DOI: 10.1016/j.fsi.2018.11.017.

17. Kewcharoen W, Srisapoome P. Probiotic effects of Bacillus spp. from Pacific white shrimp (Litopenaeus vannamei) on water quality and shrimp growth, immune responses, and resistance to Vibrio parahaemolyticus (AHPND strains). Fish and Shellfish Immunology. 2019, 94, 175189. DOI: 10.1016/j.fsi.2019.09.013. 
18. Li SW, Chen YS, Lee YS, Yang CH, Srionnual S, Wu HC, Chang CH. Comparative genomic analysis of bacteriocin-producing Weissella cibaria 110. Applied Microbiology and Biotechnology. 2017, 101(3), 1227-1237. DOI: 10.1007/s00253-016-8073-8.

19. Srionnual S, Yanagida F, Lin LH, Hsiao KN, Chen YS. Weissellicin 110, a newly discovered bacteriocin from Weissella cibaria 110, isolated from plaa-som, a fermented fish product from Thailand. Applied and Environmental Microbiology. 2007, 73(7), 2247-2450. DOI: 10.1128/ AEM.02484-06.

20. Lim HS, Yeu JE, Hong SP, Kang MS. Characterization of antibacterial cell-free supernatant from oral care probioticWeissella cibaria, CMU. Molecules: a Journal of Synthetic Chemistry and Natural Product Chemistry. 2018, 23(8), 1984. DOI: 10.3390/molecules23081984.

21. Kim M, Nam DG, Kim SB, Im P, Choe JS, Choi AJ. Enhancement of viability, acid, and bile tolerance and accelerated stability in lyophilized Weissella cibaria JW15 with protective agents. Food Science and Nutrition. 2018, 6(7), 1904-1913. DOI: 10.1002/fsn3.762.

22. Kariyawasam K, Jeewanthi RKC, Lee NK, Paik HD. Characterization of cottage cheese using Weissella cibaria D30: Physicochemical, antioxidant, and antilisterial properties. Journal of Dairy Science. 2019, 102(5), 3887-3893. DOI: 10.3168/jds.2018-15360.

23. Liu Z, Peng Z, Huang T, Guan Q, Li J, Xie M, Xiong T. Bacterial community dynamics and physicochemical characteristics in natural fermentation of jiang-shui, a traditional food made in northwest China. Journal of the Science of Food and Agriculture. 2019, 99(7), 3391-3397. DOI: 10.1002/jsfa.9556.

24. Dalmacio LM, Angeles AK, Larcia LL, Balolong MP, Estacio RC. Assessment of bacterial diversity in selected Philippine fermented food products through PCR-DGGE. Beneficial Microbes. 2011, 2(4), 273-281. DOI: 10.3920/BM2011.0017.

25. Bao R, Liu S, Ji C, Liang H, Yang S, Yan X, et al. Shortening fermentation period and quality improvement of fermented fish, Chouguiyu, by co-inoculation of Lactococcus lactis M10 and Weissella cibaria M3. Frontiers in Microbiology. 2018, 9, 3003. DOI: 10.3389/fmicb.2018.03003.

26. Yu HS, Lee NK, Choi AJ, Choe JS, Bae CH, Paik HD. Antagonistic and antioxidant effect of probiotic Weissella cibaria JW15. Food Science and Biotechnology. 2019, 28(3), 851-855. DOI: 10.1007/s10068-018-0519-6.

27. Tagg JR, McGiven AR. Assay system for bacteriocins. Applied Microbiology. 1971, 21(5), 943. https://aem.asm.org/content/21/5/943

28. Iyapparaj P, Maruthiah T, Ramasubburayan R, Prakash S, Kumar C, Immanuel G, Palavesam A. Optimization of bacteriocin production by Lactobacillus sp. MSU3IR against shrimp bacterial pathogens. Aquatic Biosystems. 2013, 9(1), 12. DOI: 10.1186/2046-9063-9-12.

29. Kandler O, Weiss N. Genus Lactobacillus Beijerinck 1901, 212AL. In: Bergey's manual of systematic bacteriology. H.A. Sneath, N.S. Mair, M.E. Sharpe, J.G. Holt (ed.). Williams and Wilkins Publ.: USA. 1986; 1209-1234.

30. Sambrook S, Russell DW. Molecular cloning: a laboratory manual. 3rd edn. Cold Spring Harbor: New York. 2003.

31. Kumar S, Stecher G, Li M, Knyaz C, Tamura K. MEGA X: Molecular evolutionary genetics analysis across computing platforms. Molecular Biology and Evolution. 2018, 35(6), 1547-1549. DOI: $10.1093 / \mathrm{molbev} / \mathrm{msy} 096$.

32. Kang MS, Yeu JE, Hong SP. Safety evaluation of oral care probiotics Weissella cibaria CMU and CMS1 by phenotypic and genotypic analysis. International Journal of Molecular Sciences. 2019, 20, 2693. DOI: 10.3390/ijms20112693.

33. Amoah K, Huang QC, Tan BP, Zhang S, Chi SY, Yang QH, Liu HY, Dong XH. Dietary supplementation of probiotic Bacillus coagulans ATCC 7050, improves the growth performance, 
intestinal morphology, microflora, immune response, and disease confrontation of Pacific white shrimp, Litopenaeus vannamei. Fish and Shellfish Immunology. 2019, 87, 796-808. DOI: 10.1016/j.fsi.2019.02.029.

34. Sha Y, Liu M, Wang B, Jiang K, Qi C, Wang L. Bacterial population in intestines of Litopenaeus vannamei fed different probiotics or probiotic supernatant. Journal of Microbiology and Biotechnology. 2016, 26(10), 1736-1745. DOI: 10.4014/jmb.1603.03078.

35. Rattanachuay P, Kantachote D, Tantirungkij M, Nitoda T, Kanzaki H. Inhibition of shrimp pathogenic vibrios by extracellular compounds from a proteolytic bacterium Pseudomonas sp. W3. Electronic Journal of Biotechnology. 2010, 13(1), 1-11. DOI: 10.2225/vol13-issue1-fulltext-2.

36. Martinez Cruz P, Ibanez AL, Monroy Hermosillo OA, Ramirez Saad HC. Use of probiotics in aquaculture. ISRN Microbiology. 2012, 2012, 916845. DOI: 10.5402/2012/916845.

37. Liu Z, Qiuqian L, Yao Z, Wang X, Huang L, Zheng J, Wang K, Li L, Zhang D. Effects of a commercial microbial agent on the bacterial communities in shrimp culture system. Frontiers in Microbiology. 2018, 9, 2430. DOI: 10.3389/fmicb.2018.02430.

38. Park HE, Kang KW, Kim BS, Lee SM, Lee WK. Immunomodulatory potential of Weissella cibaria in aged C57BL/6J mice. Journal of Microbiology and Biotechnology. 2017, 27(12), 20942103. DOI: $10.4014 / \mathrm{jmb} .1708 .08016$.

39. Wang L, Si W, Xue H, Zhao X. A fibronectin-binding protein (FbpA) of Weissella cibaria inhibits colonization and infection of Staphylococcus aureus in mammary glands. Cellular Microbiology. 2017, 19(8), e12731. DOI: 10.1111/cmi.12731. 